\title{
THE OPTICAL METHOD OF DETERMINING THE POINT SYMMETRIES
}

\author{
S. Malinowski \\ Institute of Physics, University of Łódź, Pomorska 149/153, 90-236 Łódź, Poland
}

(Received July 8, 1991)

\begin{abstract}
The present symmetry theory of optical phenomena makes it possible to determine each of 122 point symmetry groups both for non-dispersive as well as dispersive media. This is achieved by considering the symmetry breaking effect external fields (separately $\boldsymbol{E}$ and $\boldsymbol{H}$ ) on the propagation of electromagnetic waves. The corresponding results are collected in Tables I-III. The way of measuring the relevant physical quantities is proposed. The point symmetry of a given sample is obtained by comparing the experimental results with their theoretical counterparts. The results given in Tables I-III can also be viewed as the discussion of the "canonical" solutions (effects) for a given symmetry $K$.
\end{abstract}

PACS numbers: $61.16 .-\mathrm{d}, 78.20 . \mathrm{Bh}, 78.20 . \mathrm{Jq}, 78.20 . \mathrm{Ls}$

\section{Introduction}

The aim of this paper is to formulate the theoretical basis to elaborate the optical method of determining the point symmetry groups both for magnetic and non-magnetic crystals, i.e. of 122 point groups [1]. The basis is here the characteristic ("canonical") solutions of propagation equation for plane electromagnetic wave in homogenous media [2]:

$$
\left[\tilde{n}^{2}\left(\delta_{i j}-s_{i} s_{j}\right)-\dot{\tilde{\varepsilon}}_{i j}\right] E_{j}=0 \quad(i, j=1,2,3),
$$

where

$$
\tilde{n}=n+\mathrm{i} \kappa, \quad s=k / k, \quad \tilde{\varepsilon}_{i j}=\varepsilon_{i j}+i \varepsilon^{\prime}{ }_{i j} ;
$$

$\boldsymbol{E}$ stands for the electric field of the wave, $n$ is a refractive index, $\kappa-$ a damping coefficient of the wave, $k-a$ wave vector, and finally $\tilde{\varepsilon}_{i j}$ is the dielectric permittivity tensor with the complex elements.

The solution to this equation for a given $s$ depends on: 
- the form of the tensor $\tilde{\varepsilon}_{i j}$. The latter is fixed by the symmetry group $K$ of the medium or by the broken symmetry group $K(F)$ [3], if the medium is placed in external field $\boldsymbol{F}$;

- the relation between the elements of $\varepsilon$-tensor.

The solutions for the different media with the same symmetry groups $K$ (then also $K(F)$ ) can take different forms. For example: for the same form of $\tilde{\varepsilon}_{i j}$ we might obtain the elliptically or linearly polarized $E_{1}$ and $E_{2}$ waves if, respectively, $\varepsilon_{11}-\varepsilon_{22} \ll \varepsilon_{12} \ll \varepsilon_{12}^{\prime}$ or $\varepsilon_{11}-\varepsilon_{22} \ll \varepsilon_{12} \gg \varepsilon^{\prime}{ }_{12}$ hold. The question concerning the elliptical or linear character of the polarization as well as the similar problems related to Eqs. (3) and (4) of the next Section can be easily answered by taking into account the results obtained for all directions of the fields and wave vectors. However, due to the qualitative character of the results obtained in this way they do not provide a firm basis for solving the inverse problem: to determine the symmetry $K$ from the optical measurements.

In the sequel we shall make use of the solutions which either determine unambiguously the symmetry of $\tilde{\varepsilon}_{i j}$ or unambiguously exclude some possibilities, for example - the circularly polarized waves. The classical example of such solutions are the solutions for the non-magnetic crystals (32 crystallographic classes). They allowed to classify the non-magnetic crystals as the so-called biaxial, uniaxial and isotropic ones $[4,5]$.

If we apply Eq. (1) to the case of magnetic crystals (i.e. we take the form of $\varepsilon$ 's suitable for this case) we obtain new solutions. However, the number of symmetries to identify is now $90+32=122$. The way out of this situation is to supply ourselves with the sufficient number of the different forms of the tensor $\tilde{\varepsilon}_{i j}$; this can be achieved by breaking initial symmetry with the help of external field. The relevant results for $\tilde{\varepsilon}_{i j}$ were given in Tables II and III in the paper [3]. Therefore, it remains to analyse the solutions of Eq. (1) which correspond to the above tensors and to determine the symmetry group using, in turn, table I of Ref. [3]. This is accomplished in the present paper, see Tables I-III.

The proposed method is very flexible; by adjusting properly the external field we can achieve the optimal conditions. Moreover, it allows to fix experimentally the functional field dependence of the roots of Eq. (1) as well as to fix the number and the arrangement of the directions equivalent to the given direction of the field. These directions, in turn, are arranged in such a way that they are permuted under the action of the group $K$. We say they constitute the star of field $F$ in group $K$. Applying succesively the same field $\boldsymbol{F}$ parallelly to the rays of the star we obtain exactly the same effects described by the same parameters.

The corresponding results are presented in Tables I-III. The following notation is used in these tables: $\boldsymbol{F}=0$ denotes non-dispersive crystal without external field, $\boldsymbol{F}=\boldsymbol{k}-$ a dispersive crystal, while $\boldsymbol{F}=\boldsymbol{E}, \boldsymbol{H}$ - any crystal in the external electric or magnetic field, respectively. Other symbols are explained in Sec. 2. In Sec. 3 some suggestions are given concerning the measurement of the "canonical". properties of the crystal. 


\section{The propagation equation and the symmetry of its solutions}

It follows from Eq. (1) that (if $\operatorname{det}\left(\tilde{\varepsilon}_{i j}\right) \neq 0$ ) the electric fields $\boldsymbol{E}_{1}$ and $\boldsymbol{E}_{2}$ of electromagnetic waves are oriented with respect to the vector $s$ in such a way,
that we have:

(a) two skew waves,

(b) a skew and transverse wave, or

(c) two transverse waves [6].

In the case (c), for some forms of the tensors $\tilde{\varepsilon}_{i j}$, the classification can be made more detailed to include the linearly, $\mathrm{L}$, and circilarly, C, polarized waves; we use the symbols: $\mathrm{cL}, \mathrm{cC}, \mathrm{c}$. The latter symbol denotes the waves for which the circular polarization is forbidden.

Equation (1) is invariant under the operation of the inversion of the direction field, $\boldsymbol{F} \rightarrow-\boldsymbol{F}$, if the tensor $\tilde{\varepsilon}_{i j}$ determined by the $K(\boldsymbol{F})$ groups and is not invariant if it is determined by the $J(F)$ groups [2, 3]. As a consequence the roots of Eq. (1) must satisfy, in the case of $K(F)$ groups, the following relation:

$$
\tilde{n}_{1,2}^{2}( \pm F)=\tilde{n}_{1,2}^{2}(\mp F)
$$

or

$$
\tilde{n}_{1,2}^{2}( \pm F)=\tilde{n}_{2,1}^{2}(\mp F),
$$

while in the case of $J(F)$ groups neither (3) nor (4) can hold, i.e.

$$
\tilde{n}_{1,2}^{2}( \pm F) \neq \tilde{n}_{1,2}^{2}(\mp F), \quad \tilde{n}_{1,2}^{2}( \pm F) \neq \tilde{n}_{2,1}^{2}(\mp F) .
$$

Therefore, the information concerning the type of the wave may be supplied with the additionalinformation,relatedtoEqs.(3)-(5). For example, we can use the symbols: c3, c4, c5, b3, b5, a3, a5, c, b, a, while if there is no additional number it means that Eq. (5) cannot be obeyed and we cannot decide which one of Eqs.(3) and (4) holds.

In some cases of linearly polarized waves, the conditions (3) and (4) for the birefringence give

$$
\Delta n=0,
$$

while in some cases of elliptically and circularly polarized waves they give for the angle of rotation of polarization plane

$$
\varphi(F)=\varphi(-F) \quad(\text { cp.with (3)) }
$$

or

$$
\varphi(\boldsymbol{F})=-\varphi(-F) \quad(\text { cp.with (4)). }
$$

The rotations described by Eqs. (7) and (8) will be called the non-reciprocal and reciprocal rotation, respectively. 

relation

The condition (5) does not exclude, for some linearly polarized waves, the $\Delta n=0$

identical, from mathematical point of view with the formula (6); however, they are different as far as the measurement is concerned. The formulae (7) and (8) do not have counterparts for the elliptically and circularly polarized waves, because here

$$
\varphi(\boldsymbol{F}) \neq \varphi(-\boldsymbol{F}) \text { and also } \varphi(\boldsymbol{F}) \neq-\varphi(-\boldsymbol{F}) \text {. }
$$

As a consequence, for the groups $J(F)$ the pure non-reciprocal and reciprocal rotation do not exist.

Summerizing, we have the new symbols: cC5, cL6, cC7, cC8 and cL9.

When $\boldsymbol{F}=0$ the symbols a, b, c, cL, cC are sufficient to describe the solutions of Eq. (1). Additional informations concerning $\Delta n(M)$ and $\varphi(M)(M$ is the magnetization vector of the magnetic domain of the symmetry $K$ ) are trivial; therefore, they are neglected in Tables I-III. The solutions of type cC (and similarly for the elliptically polarized waves) describe the Faraday effect generated by the (structural) vector $M$ of crystal. This effect is known as the extraordinary Faraday effect. We propose the name: the structural Faraday effect. To motivate this, let us take into account the following facts: the solutions of Eq. (1) for the structures with the symmetry $K=3 m^{\prime}$, i.e. $M \neq 0$, are of the type $\mathrm{cC}$, while for the structures with the symmetry $K=3 m 1^{\prime}$, i.e. $M=0$, they are of the type cL (see: Table II).

To summarize, if $\boldsymbol{F} \neq 0$ we obtain the same solutions of Eq. (1) for each ray of $\boldsymbol{F}$-star. Finally, the full symbols of solutions look like below:

(i) a3,6 denotes two skew waves for which $\tilde{n}_{1}^{2}(F)$ and $\tilde{n}_{2}^{2}(F)$ satisfy the relation (3) and $\boldsymbol{F}$-star has 6 rays;

(ii) a,4 denotes two skew waves; the relation (3) or (4) (but not (5)) holds and the $F$-star has 4 rays;

(iii) $\mathrm{cC} 7,2$ denotes the non-reciprocal rotation of circularly polarized transverse waves; $\boldsymbol{F}$-star has 2 rays; and so on.

\section{The tables and measurements}

Table I concerns the biaxial crystals, Table II - uniaxial crystals and Table III - isotropic (cubic) crystals. If a sample appears to be magnetized $(M \neq 0)$ solutions should be looked for under No 1-12 (Table I) and 29-47 (Table II); otherwise $(M=0)$ - under No 13-28 (Table I) and 48-106 (Table II).

For definiteness from now we will consider the case of external electric field $\boldsymbol{E}$; the requirements concerning the geometry of both $\boldsymbol{E}$ and $\boldsymbol{H}$ field are exactly the same.

At the beginning let us suppose that our sample belongs to the uniaxial crystal (Table II - No $29-47$ for $M \neq 0$, No $48-106$ for $M=0$ ). It is convenient to perform the first measurement in the geometry $s\|E\| z$ and $s\|z, E\|-z$, for two opposite directions of the field $\boldsymbol{E}$. The direction of $s$ must be preserved, 
otherwise it is not possible to recognize whether angle $\varphi(E)$ satisfies the relation (7) or the relation (8). In the above geometry the solution of type cC5 can also be recognized, because, in general, the requirements concerning the geometry for the groups $J(F)$ are weaker than for the groups $K(F)$. In the case of solutions of type $\mathrm{CL}$ for both above geometries and both type of groups (i.e. $J(\boldsymbol{F})$ and $K(\boldsymbol{F})$ ) we obtain $\Delta n=0$. In order to distinguish them (i.e. which is of the type cL6 and which of type $\mathrm{CL} 9$ ) the additional measurements (at best - in the geometries $s \perp z, E \| z$ and $s \perp z, E \|-z)$ are needed.

Of course, for a given sample we can obtain only one from eight (namely: $c, 2$; $\mathrm{c4}, 2 ; \mathrm{cC} 5,1 ; \mathrm{cL} 4,2 ; \mathrm{cL} 6,2 ; \mathrm{cC} 7,2 ; \mathrm{cC} 8,2 ; \mathrm{cL} 9,1)$ possible results. The above results are written out for each groups $K$ in the first line in Table II. This line is equipped with the symbol $\boldsymbol{F} \| \boldsymbol{z}$.

The eight effects for 19 (for $M \neq 0$ ) +59 (for $M=0$ ) groups of the uniaxial crystals are not sufficient to determine the symmetry of a given sample; the subsequent measurements are unavoidable. First of all we should perform the additional measurements for $F \perp z$ because only on this plane there exist more than one qualitatively different $\boldsymbol{F}$-stars. In Table II the theoretical results for the case $F \perp z$ are listed successively, starting from the second line, in the order of decreasing symmetry of $\boldsymbol{F}$-stars; all results are given for the geometry $s \| \boldsymbol{F} \perp z$.

There exists a continuum of lowest symmetry $\boldsymbol{F}$-stars, while there are only one or two stars for any higher symmetry. All stars from the continuum have, of course, the same symmetry; the solutions corresponding to all of them are of the same type (a) or sometimes (b) - see Table II, they have the same number of rays; nevertheless, and this is what is more important, for the same value of the field $\boldsymbol{F}$ the values for $\tilde{n}_{1}^{2}(\boldsymbol{F})$ and also for $\tilde{n}_{2}^{2}(\boldsymbol{F})$ can be different (more precisely: not much different, but still different).

The latter property suggests the method of measurement. The ideal would be the permanent measurement during the slow rotation of the crystal, or at least, the successive measurements for the crystal rotated by the very small angle around $z$-axis until the full $2 \pi$ angle was achieved. It is better to depict the measured values of $\tilde{n}_{1}^{2}$ and $\tilde{n}_{2}^{2}$ (for the same value of the field $F$ ) in the polar coordinates. In this way we can easily find the number of rays of a given star. The corresponding curves will be very irregular and closed (if for a given value of the field $\boldsymbol{F}$ there exists no direction along which the field $\boldsymbol{F}$ causes the phase transition). The irregularities of the curves are controlled by the rules of symmetry $K$. To be more precise: such curves must have the symmetry of two-dimensional (the symmetry group of the plane under consideration) subgroup of $K$-group. The irregularity comes from the experimental errors. Such properties are to be expected if we remind ourselves the corresponding measurements for the so-called "Umkehreffekt" for transport phenomena ([7], and the literature therein). To summarize, the method of determining the number of rays in $\boldsymbol{F}$-star proposed above, seems to be the only one available.

If to the star from the continuum there correspond the type (a) waves then to the single star there correspond the type (b) or (c) ones. The problem of distinguishing between them reduces to the problem of distinguishing between the transverse and skew waves. It is known [6] that under the same conditions the skew 
wave is more absorbed than transverse one. At the point in which the type (c) wave (type (b)) transforms into the type (b) one (type (a)) the curve of damping coefficient $\kappa$ must receive a jump.

By comparing the results of the experiments proposed above with the theoretical data (see Table II) one can reduce the ambiguity in determining the symmetry group of the crystal to a few possibilities only. Then, the final answer can be obtained by performing in general one additional experiment with the external magnetic field.

There are also some cases (e.g. Table II No 51, 52) that the measurements for $\boldsymbol{F}=\boldsymbol{E}, \boldsymbol{H}$ arbitrarily oriented (a.o.) with respect to the $z$-axis of the crystals are needed. In such a case we give in Table I-II (the extra line, denoted by symbol a.o) the full information about those $\boldsymbol{F}$-stars. However, in order to recognize such symmetries one measurement along only one $s$ direction for the geometry $s \| F$ (and, of course, for $s \|-F$ ) is sufficient.

In order to determine the symmetry of isotropic (cubic) crystal one needs twice as many measurements; they have to be performed along the edge (and perpendicular plane) of the cube and along the diagonal (and perpendicular plane) of it. In Table III it is assumed that the direction of the cube edge is known from other experiment. This is because it is troublesome to find this directions by optical methods, in particular in non-dispersive media. This problem will be considered elsewhere.

There is no problem in the case of biaxial (Table I) crystals. The $\mathrm{C}_{2 z}$ axis of crystal (if it exists) is parallel or perpendicular to the bisector of the angle made by optic axes.

\section{Summary}

In Tables I-III the results concerning the "canonical" effects for dispersive media $(F=k)$ are given. They allow to solve the question whether our medium is dispersive or not. If it is dispersive - the advantage of the proposed method is that, by varying the external fields, one can isolate the contributions coming from the external field as well as those related to the dispersive character of the medium and, therefore, determine unambiguously (as contrasted with the previous proposals for dispersive media [8]) the symmetry of the crystal.

The further problem is to translate the theoretical proposition into the experimental methods. It is most likely that some experimental methods presented in Ref. [9] (and the literature therein) and Ref. [10] (and the literature therein) may be adopted to our problem.

The above results can be also considered as:

- the discussion of the cardinal solutions (effects) for a given symmetry $K$,

- the proposal of looking for the other cardinal solutions for other geometries, as for example $s \| z$ and $F \perp z$ or $s \perp F \perp z$, and so on, for a given symmetry $K$. 
TABLE I

The characteristic optical effects of the biaxial crystals in the fields $\boldsymbol{F}=\boldsymbol{k}, \boldsymbol{E}, \boldsymbol{H}$. (For abbreviations see the text).

\begin{tabular}{|c|c|c|c|c|c|c|c|}
\hline \multirow[b]{2}{*}{ No } & \multirow[b]{2}{*}{$K$} & \multirow[b]{2}{*}{$M$} & \multirow[b]{2}{*}{$s$} & \multicolumn{4}{|c|}{ The effects for $\boldsymbol{F}=$} \\
\hline & & & & 0 & $\boldsymbol{k}$ & $\boldsymbol{E}$ & $\boldsymbol{H}$ \\
\hline$(1)$ & $(2)$ & (3) & $(4)$ & (5) & $(6)$ & $(7)$ & $(8)$ \\
\hline 1 & 1 & a.o. & a.o. & $\mathrm{a}$ & $\mathbf{a} 5,1$ & $\mathrm{a} 5,1$ & $\mathrm{a} 5,1$ \\
\hline 2 & $\overline{1}$ & a.o. & a.o. & $\mathrm{a}$ & a3,2 & a3,2 & $\mathrm{a} 5,1$ \\
\hline \multirow[t]{2}{*}{3} & $2^{\prime}$ & $\perp z$ & $\|$ & $\mathrm{a}$ & a,2 & a5,1 & $\mathrm{a}, 2$ \\
\hline & & & $\perp$ & a & a5,1 & ă,2 & a5,1 \\
\hline \multirow[t]{2}{*}{4} & $m$ & $\perp z$ & $\|$ & a & a5,1 & $a, 2$ & $\mathrm{a}, 2$ \\
\hline & & & $\perp$ & a & a, 2 & a5,1 & a5,1 \\
\hline \multirow[t]{2}{*}{5} & $2^{\prime} / m^{\prime}$ & $\perp z$ & $\|$ & $\mathrm{a}$ & a3,2 & a3,2 & $a, 2$ \\
\hline & & & $\perp$ & a & a3,2 & a3,2 & $\mathrm{a} 5,1$ \\
\hline \multirow[t]{2}{*}{6} & $m$ & $\| z$ & $\|$ & c & a,2 & $a, 2$ & $\mathrm{a}, 2$ \\
\hline & & & $\perp$ & b & b5, 1 & b5,1 & $\mathrm{a}, 2$ \\
\hline \multirow[t]{2}{*}{7} & 2 & $\| z$ & $\|$ & c & $\mathrm{c} 5,1$ & $\mathrm{c} 5,1$ & $c 5,1$ \\
\hline & & & $\perp$ & b & $\mathrm{a}, 2$ & $a, 2$ & a, 2 \\
\hline \multirow[t]{2}{*}{8} & $2 / m$ & $\| z$ & $\|$ & c & $\mathrm{c} 3,2$ & $\mathrm{c} 3,2$ & $\mathrm{c} 5,1$ \\
\hline & & & $\perp$ & b & $\mathrm{b} 3,2$ & b3,2 & $a, 2$ \\
\hline \multirow[t]{4}{*}{9} & $2{ }^{\prime}{ }^{\prime} 2$ & $\| z$ & $\|$ & c & c5 5,1 & $\mathrm{c}, 2$ & c5,1 \\
\hline & & & $\ddot{\perp}$ & $\mathrm{b}$ & $\mathrm{a}, 2$ & $\mathrm{a}, 2$ & $\mathbf{a}, 2$ \\
\hline & & & & b & $\mathrm{a}, 2$ & $\mathrm{a}, 2$ & $\mathrm{a}, 2$ \\
\hline & & & & b & $a, 4$ & $a, 4$ & $\mathrm{a}, 4$ \\
\hline \multirow[t]{4}{*}{10} & m'm'2 & $\| z$ & $\|$ & c & $c, 2$ & c5,1 & c5,1 \\
\hline & & & $\perp$ & b & $\mathrm{a}, 2$ & a,2 & $\mathrm{a}, 2$ \\
\hline & & & & b & a,2 & $a, 2$ & $a, 2$ \\
\hline & & & & b & a,4 & $\mathrm{a}, 4$ & $\mathrm{a}, 4$ \\
\hline \multirow[t]{4}{*}{11} & m'm'm & $\| z$ & $\|$ & c & $\mathrm{c} 3,2$ & $\mathrm{c} 3,2$ & c5,1 \\
\hline & & & $\perp$ & b & $\mathrm{b} 3,2$ & $\mathrm{~b} 3,2$ & $\mathrm{a}, 2$ \\
\hline & & & & b & $\mathrm{b} 3,2$ & b3,2 & $a, 2$ \\
\hline & & & & b & b3,4 & b3,4 & $a, 4$ \\
\hline \multirow[t]{4}{*}{12} & m'm2' & $\| y$ & $\|$ & b & b3,2 & b5,1 & $\mathrm{a}, 2$ \\
\hline & & & $\perp$ & b & b5, 1 & b3,2 & $\mathrm{a}, 2$ \\
\hline & & & & $\mathbf{a}$ & a,2 & $a, 2$ & c5,1 \\
\hline & & & & a & a5,2 & $\mathrm{a}, 4$ & a5,2 \\
\hline 13 & $\overline{1}$ & & a.o. & a & a5, 1 & $\mathrm{a}, 2$ & a,2 \\
\hline 14 & $11^{\prime}$ & & a.o. & $\mathbf{a}$ & $a, 2$ & a 5,1 & a, 2 \\
\hline 15 & $\overline{1} 11^{\prime}$ & & a.o. & $a$ & a3,2 & a3,2 & $\mathrm{a}, 2$ \\
\hline \multirow[t]{2}{*}{16} & $m 1^{\prime}$ & & $\|$ & c & $\mathrm{a}, 2$ & a,2 & $\mathrm{c}, 2$ \\
\hline & & & $\perp$ & b & b3,2 & b5,1 & $a, 2$ \\
\hline \multirow[t]{2}{*}{17} & $2^{\prime} / m$ & & $\|$ & c & $\mathrm{a}, 2$ & $a, 2$ & $\mathrm{c}, 2$ \\
\hline & & & $\stackrel{\perp}{\perp}$ & b & b5,1 & b3,2 & $\mathrm{a}, 2$ \\
\hline \multirow[t]{2}{*}{18} & $21^{\prime}$ & & $\|$ & c & c, 2 & $\mathrm{c} 5,1$ & $c, 2$ \\
\hline & & & $\perp$ & b & $a, 2$ & a,2 & $a, 2$ \\
\hline
\end{tabular}


TABLE I (cont.)

\begin{tabular}{|c|c|c|c|c|c|c|}
\hline$\overline{(1)}$ & (2) & (4) & $\overline{\overline{(5)}}$ & (6) & (7) & $(8)$ \\
\hline \multirow[t]{2}{*}{19} & $2 / m^{\prime}$ & $\pi$ & $\mathrm{c}$ & $\mathrm{c5}, 1$ & $c, 2$ & $c, 2$ \\
\hline & & $\perp$ & b & a,2 & $a, 2$ & $a, 2$ \\
\hline \multirow[t]{2}{*}{20} & $2 / m 1^{\prime}$ & $\|$ & c & c3,2 & $c 3,2$ & c,2 \\
\hline & & 1 & b & b3,2 & b3,2 & $\mathrm{a}, 2$ \\
\hline \multirow[t]{5}{*}{21} & 222 & $\|$ & $\mathrm{cL}$ & $c, 2$ & $c, 2$ & $c, 2$ \\
\hline & & $\perp$ & cL & $c, 2$ & c, 2 & $c, 2$ \\
\hline & & & $\mathrm{cL}$ & $c, 2$ & c, 2 & $c, 2$ \\
\hline & & & b & $\mathrm{a}, 4$ & $a, 4$ & $a, 4$ \\
\hline & & a.o. & $\mathbf{a}$ & $\mathrm{a} 5,4$ & a5,4 & a5,4 \\
\hline \multirow[t]{5}{*}{22} & 2221 ' & $\|$ & cL & $c, 2$ & c,2 & $c, 2$ \\
\hline & & 1 & $\mathrm{cL}$ & c,2 & c,2 & c,2 \\
\hline & & & cL & c, 2 & c, 2 & $c, 2$ \\
\hline & & & b & $a, 4$ & $a, 4$ & $a, 4$ \\
\hline & & a.o. & $\mathbf{a}$ & $a, 8$ & a5,4 & a,8 \\
\hline \multirow[t]{5}{*}{23} & $m^{\prime} m^{\prime} m^{\prime}$ & $\|$ & cL & $c, 2$ & c, 2 & $c, 2$ \\
\hline & & $\perp$ & cL & $c, 2$ & $c, 2$ & $c, 2$ \\
\hline & & & cL & $c, 2$ & $c, 2$ & $c, 2$ \\
\hline & & & b & $a, 4$ & $\mathrm{a}, 4$ & $a, 4$ \\
\hline & & a.o. & a & a5,4 & $a, 8$ & $a, 8$ \\
\hline \multirow[t]{5}{*}{24} & $m m 2$ & $\|$ & $\mathrm{cL}$ & cL5,1 & cL5,1 & $c, 2$ \\
\hline & & 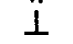 & cL & b3,2 & b3,2 & $c, 2$ \\
\hline & & & cL & b3,2 & b3,2 & $c, 2$ \\
\hline & & & b & $a, 4$ & $a, 4$ & $\mathrm{a}, 4$ \\
\hline & & a.o. & a & a5,4 & a 5,4 & a5,4 \\
\hline \multirow[t]{5}{*}{25} & $m m 21$ & $\|$ & cL & $\mathrm{cL} 3,2$ & cL5,1 & $c, 2$ \\
\hline & & $\stackrel{1}{\perp}$ & $\mathrm{cL}$ & b3,2 & b3,2 & c, 2 \\
\hline & & & $\mathrm{cL}$ & b3,2 & b3,2 & c,2 \\
\hline & & & $\mathrm{b}$ & a,4 & $a, 4$ & $a, 4$ \\
\hline & & a.o. & a & $a, 8$ & a5,4 & $a, 8$ \\
\hline \multirow[t]{5}{*}{26} & $m m m$ & $\|$ & $\mathrm{cL}$ & $\mathrm{cL} 3,2$ & cL3,2 & $c, 2$ \\
\hline & & $\perp$ & cL & cL3,2 & cL3,2 & $c, 2$ \\
\hline & & & cL & cL3,2 & cL3,2 & $c, 2$ \\
\hline & & & $\mathrm{b}$ & b3,4 & b3 3,4 & $\mathrm{a}, 4$ \\
\hline & & a.o. & a & a3,8 & $\mathrm{a} 3,8$ & $\mathrm{a} 5,4$ \\
\hline \multirow[t]{5}{*}{27} & $m m m 1^{\prime}$ & $\|$ & $\mathrm{cL}$ & cL3,2 & cL3,2 & $c, 2$ \\
\hline & & $\perp$ & $\mathrm{cL}$ & cL3,2 & cL3,2 & $\mathrm{c}, 2$ \\
\hline & & & $\mathrm{cL}$ & cL3,2 & cL3,2 & $c, 2$ \\
\hline & & & b & b3,4 & b3,4 & $a, 4$ \\
\hline & & a.o. & $\mathbf{a}$ & a3,8 & a3,8 & $a, 8$ \\
\hline \multirow[t]{4}{*}{28} & $m m m^{\prime}$ & $\|$ & $\mathrm{cL}$ & cL5,1 & cL3,2 & $c, 2$ \\
\hline & & $\ddot{\perp}$ & $\mathrm{cL}$ & b3,2 & b3,2 & c, 2 \\
\hline & & & cL & b3,2 & b3,2 & $\mathrm{c}, 2$ \\
\hline & & & , & $a, 4$ & $a, 4$ & $a, 4$ \\
\hline
\end{tabular}


TABLE II

The characteristic optical effects of the uniaxial crystals in the fields $\boldsymbol{F}=\boldsymbol{k}, \boldsymbol{E}, \boldsymbol{H}$. (For abbreviations see the text).

\begin{tabular}{|c|c|c|c|c|c|c|c|}
\hline \multirow[b]{2}{*}{ No } & \multirow[b]{2}{*}{$K$} & \multirow[b]{2}{*}{$M$} & \multirow[b]{2}{*}{$\boldsymbol{s}$} & \multicolumn{4}{|c|}{ The effects for $F=$} \\
\hline & & & & 0 & $k$ & $\boldsymbol{E}$ & $\boldsymbol{H}$ \\
\hline (1) & (2) & (3) & (4) & (5) & (6) & (7) & (8) \\
\hline \multirow[t]{2}{*}{29} & $\overline{4}$ & $\| z$ & $\|$ & $\mathrm{cC}$ & $c, 2$ & $c, 2$ & $\mathrm{cC5}, 1$ \\
\hline & & & $\perp$ & b & $a, 4$ & $\mathrm{a}, 4$ & $\mathrm{a}, 4$ \\
\hline \multirow[t]{4}{*}{30} & $\overline{4} 2^{\prime} m^{\prime}$ & $\| z$ & $\|$ & $\mathrm{cC}$ & c,2 & $c, 2$ & cC5,1 \\
\hline & & & $\perp$ & $\mathrm{b}$ & $a, 4$ & $a, 4$ & $\mathbf{a}, 4$ \\
\hline & & & & b & $a, 4$ & $\mathrm{a}, 4$ & $a, 4$ \\
\hline & & & & b & $a, 8$ & $\mathrm{a}, 8$ & a,8 \\
\hline \multirow[t]{2}{*}{31} & 3 & $\| z$ & $\|$ & $\mathrm{cC}$ & $\mathrm{cC} 5,1$ & $\mathrm{cC5}, 1$ & cC5,1 \\
\hline & & & $\perp$ & b & $\mathbf{a} 5,3$ & a5,3 & a5,3 \\
\hline \multirow[t]{4}{*}{32} & $3 m^{\prime}$ & $\| z$ & $\|$ & $\mathrm{cC}$ & $\mathrm{cC} 7,2$ & cC5,1 & cC5,1 \\
\hline & & & $\perp$ & $\mathrm{b}$ & a5,3 & $a, 6$ & a, 6 \\
\hline & & & & b & a, 6 & $\mathrm{a} 5,3$ & $a 5,3$ \\
\hline & & & & $\mathrm{b}$ & a5,6 & a5,6 & a5,6 \\
\hline \multirow[t]{2}{*}{33} & 4 & $\| z$ & $\|$ & $\mathrm{cC}$ & $\mathrm{cC} 5,1$ & $\mathrm{cC} 5,1$ & cC5,1 \\
\hline & & & $\perp$ & $\mathrm{b}$ & $\mathrm{a}, 4$ & $a, 4$ & a,4 \\
\hline \multirow[t]{2}{*}{34} & 6 & $\| z$ & $\|$ & $\mathrm{cC}$ & cC5,1 & $\mathrm{cC} 5,1$ & $\mathrm{cC5}, 1$ \\
\hline & & & 1 & $\mathrm{~b}$ & a, 6 & $a, 6$ & $a, 6$ \\
\hline \multirow[t]{4}{*}{35} & $6 m^{\prime} m^{\prime}$ & $\| z$ & $\|$ & $\mathrm{cC}$ & $\mathrm{cC} 7,2$ & $\mathrm{cC} 5,1$ & $\mathrm{cC} 5,1$ \\
\hline & & & $\perp$ & b & a, 6 & $a, 6$ & $a, 6$ \\
\hline & & & & $\mathrm{b}$ & a, 6 & a, 6 & a,6 \\
\hline & & & & $\mathrm{b}$ & a,12 & a,12 & a,12 \\
\hline \multirow[t]{4}{*}{36} & $4 m^{\prime} m$ ' & $\| z$ & $\|$ & $\mathrm{cC}$ & $\mathrm{cC} 7,2$ & cC5,1 & $\mathrm{cC5}, 1$ \\
\hline & & & $\perp$ & $\mathrm{b}$ & $a, 4$ & $\mathrm{a}, 4$ & $a, 4$ \\
\hline & & & & b & $a, 4$ & $\mathbf{a}, 4$ & $a, 4$ \\
\hline & & & & b & $a, 8$ & $a, 8$ & $a, 8$ \\
\hline \multirow[t]{4}{*}{37} & $32^{\prime}$ & $\| z$ & $\|$ & $\mathrm{cC}$ & $\mathrm{cC5}, 1$ & $\mathrm{cC} 7,2$ & cC5,1 \\
\hline & & & $\ddot{\perp}$ & b & a, 6 & a5,3 & $\mathrm{a}, 6$ \\
\hline & & & & b & a5,3 & $\mathrm{a}, 6$ & a5,3 \\
\hline & & & & b & a5,6 & a5,6 & a5,6 \\
\hline \multirow[t]{3}{*}{38} & $42^{\prime} 2^{\prime}$ & $\| z$ & $\|$ & $\mathrm{cC}$ & $\mathrm{cC5}, 1$ & $\mathrm{cC} 7,2$ & $\mathrm{cC} 5,1$ \\
\hline & & & $\perp$ & b & $a, 4$ & $a, 4$ & $\mathrm{a}, 4$ \\
\hline & & & & b & $\mathrm{a}, 4$ & $\mathrm{a}, 4$ & $\mathrm{a}, 4$ \\
\hline \multirow{4}{*}{39} & 62'2' & $\| z$ & & D & & & $\begin{array}{r}\text { a,d } \\
\text { rat }\end{array}$ \\
\hline & & & $\underline{I}$ & b & a, 6 & a, 6 & a, 6 \\
\hline & & & & b & a,6 & $a, 6$ & $a, 6$ \\
\hline & & & & b & $\mathrm{a}, 12$ & a,12 & a,12 \\
\hline \multirow[t]{2}{*}{40} & $\overline{3}$ & $\| z$ & $\|$ & $\mathrm{cC}$ & $\mathrm{cC} 7,2$ & $\mathrm{cC} 7,2$ & $\mathrm{cC} 5,1$ \\
\hline & & & $\perp$ & h & a3,6 & a3,6 & a5,3 \\
\hline
\end{tabular}


TABLE II (cont.)

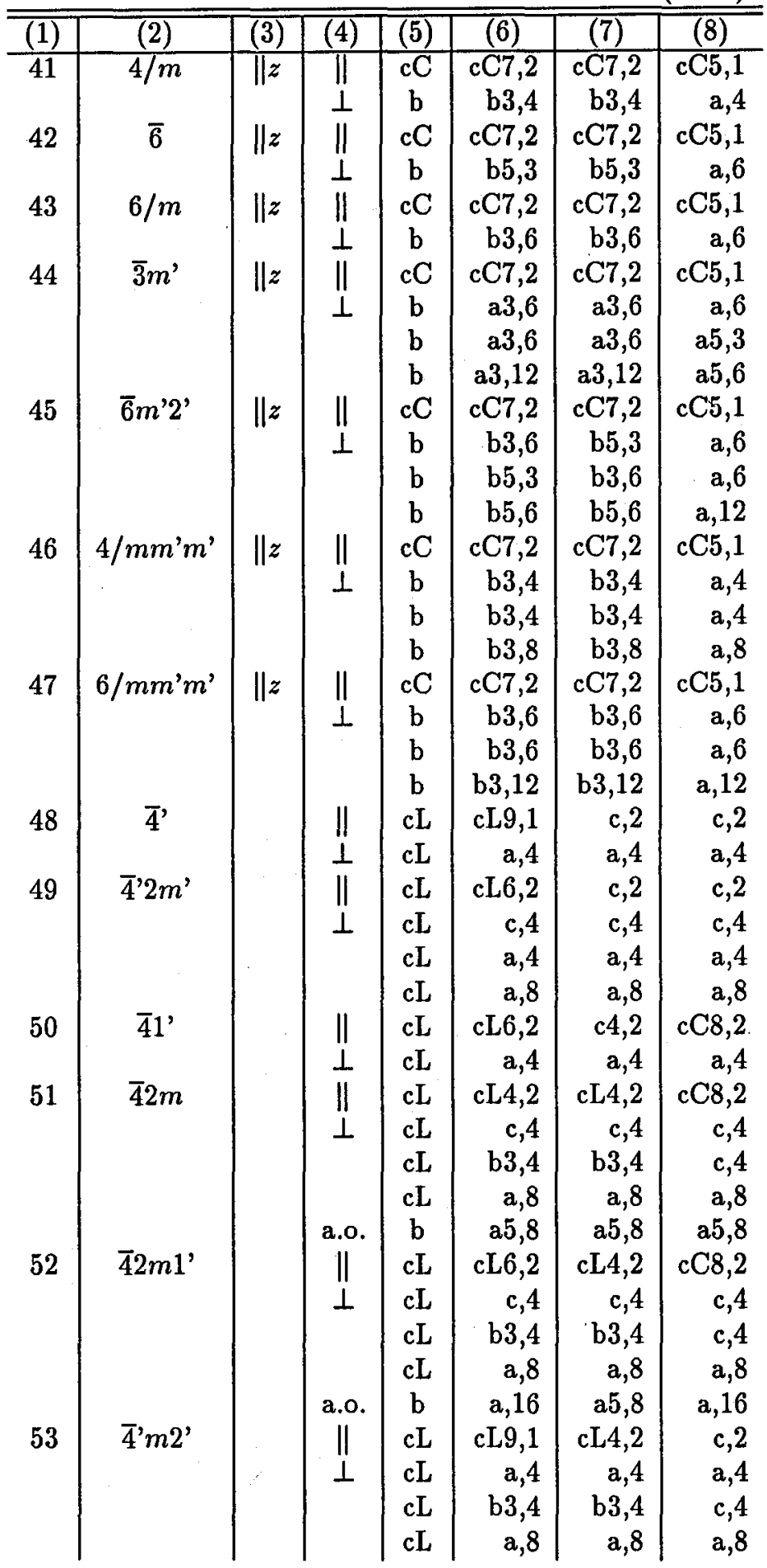


TABLE II (cont.)

\begin{tabular}{|c|c|c|c|c|c|c|}
\hline (1) & $\overline{(2)}$ & $\overline{(4)}$ & $\overline{(5)}$ & (6) & (7) & (8) \\
\hline \multirow[t]{2}{*}{54} & $\overline{3}$ & 1 & $\mathrm{cL}$ & cL9,1 & $\mathrm{cC} 8,2$ & cC8,2 \\
\hline & & $\ddot{\perp}$ & $\mathrm{cL}$ & a5,3 & $a, 6$ & $a, 6$ \\
\hline \multirow[t]{2}{*}{55} & $\overline{6}$, & $\|$ & $\mathrm{cL}$ & cL9,1 & cC8,2 & cC8,2 \\
\hline & & 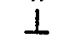 & $\mathrm{cL}$ & $a, 6$ & c5,3 & c5,3 \\
\hline \multirow[t]{2}{*}{56} & $4 / m^{\prime}$ & $\|$ & cL & cL9,1 & $\mathrm{cC} 8,2$ & cC8,2 \\
\hline & & $\ddot{L}$ & $\mathrm{cL}$ & $a, 4$ & $a, 4$ & $a, 4$ \\
\hline \multirow[t]{2}{*}{57} & $6 / m^{2}$ & $\|$ & $\mathrm{cL}$ & cL 9,1 & $\mathrm{cC} 8,2$ & cC8,2 \\
\hline & & $\perp$ & cL & $a, 6$ & $a, 6$ & $a, 6$ \\
\hline \multirow[t]{4}{*}{58} & 32 & $\|$ & $\mathrm{cL}$ & $\mathrm{cC} 8,2$ & $\mathrm{cC} 8,2$ & $\mathrm{cC} 8,2$ \\
\hline & & $\ddot{\perp}$ & $\mathrm{cL}$ & $\mathrm{c} 5,3$ & c5,3 & $\mathrm{c} 5,3$ \\
\hline & & & $\mathrm{cL}$ & $a, 6$ & $a, 6$ & $a, 6$ \\
\hline & & & cL & a5,6 & $a 5,6$ & 25,6 \\
\hline \multirow[t]{4}{*}{59} & $\overline{3}^{\prime} m^{\prime}$ & $\|$ & $\mathrm{cL}$ & cL6,2 & $\mathrm{cC} 8,2$ & cC8,2 \\
\hline & & $\stackrel{\perp}{ }$ & $\mathrm{cL}$ & c5,3 & $\mathrm{c}, 6$ & $c, 6$ \\
\hline & & & $\mathrm{cL}$ & $a, 6$ & $a, 6$ & $a, 6$ \\
\hline & & & $\mathrm{cL}$ & $\mathrm{a} 5,6$ & $a, 12$ & $a, 12$ \\
\hline \multirow[t]{4}{*}{60} & 422 & $\|$ & $\mathrm{cL}$ & $\mathrm{cC} 8,2$ & $\mathrm{cC} 8,2$ & cC8,2 \\
\hline & & $\perp$ & $\mathrm{cL}$ & $c, 4$ & $c, 4$ & $c, 4$ \\
\hline & & & cL & $\mathrm{c}, 4$ & $c, 4$ & $c, 4$ \\
\hline & & & $\mathrm{cL}$ & $a, 8$ & $a, 8$ & $a, 8$ \\
\hline \multirow[t]{4}{*}{61} & 622 & $\|$ & $\mathrm{cL}$ & $\mathrm{cC} 8,2$ & $\mathrm{cC} 8,2$ & $\mathrm{cC} 8,2$ \\
\hline & & 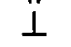 & $\mathrm{cL}$ & $c, 6$ & $c, 6$ & $c, 6$ \\
\hline & & & $\mathrm{cL}$ & $c, 6$ & $c, 6$ & $c, 6$ \\
\hline & & & $\mathrm{cL}$ & $a, 12$ & $a, 12$ & a, 12 \\
\hline \multirow[t]{4}{*}{62} & $\overline{6} ' m^{\prime} 2$ & $\|$ & $\mathrm{cL}$ & cL 6,2 & $\mathrm{cC} 8,2$ & cC8,2 \\
\hline & & $\ddot{\perp}$ & $\mathrm{cL}$ & $c, 6$ & c5,3 & c5,3 \\
\hline & & & $\mathrm{cL}$ & $a, 6$ & $a, 6$ & $a, 6$ \\
\hline & & & $\mathrm{cL}$ & $a, 12$ & $a 5,6$ & $a 5,6$ \\
\hline \multirow[t]{4}{*}{63} & $4 / m^{\prime} m^{\prime} m^{\prime}$ & $\|$ & $\mathrm{cL}$ & cL6,2 & $\mathrm{cC} 8,2$ & $\mathrm{cC} 8,2$ \\
\hline & & $\perp$ & $\mathbf{c L}$ & $c, 4$ & $c, 4$ & $c, 4$ \\
\hline & & & $\mathrm{cL}$ & $c, 4$ & $c, 4$ & $c, 4$ \\
\hline & & & $\mathrm{cL}$ & $a, 8$ & $a, 8$ & $a, 8$ \\
\hline \multirow[t]{4}{*}{64} & $6 / m^{\prime} m^{\prime} m^{\prime}$ & $\|$ & $\mathrm{cL}$ & cL6,2 & $\mathrm{cC} 8,2$ & $\mathrm{cC} 8,2$ \\
\hline & & $\perp$ & $\mathrm{cL}$ & c, 6 & $c, 6$ & $c, 6$ \\
\hline & & & $\mathrm{cL}$ & $c, 6$ & $c, 6$ & $c, 6$ \\
\hline & & & $\mathrm{cL}$ & $a, 12$ & a, 12 & $\mathrm{a}, 12$ \\
\hline \multirow[t]{2}{*}{65} & $\overline{3} 1{ }^{\prime}$ & $\|$ & $\mathrm{cL}$ & cL6,2 & cL6,2 & $\mathrm{cC} 8,2$ \\
\hline & & $\ddot{\perp}$ & $\mathrm{cL}$ & a3,6 & a3,6 & $a, 6$ \\
\hline \multirow[t]{2}{*}{66} & $6^{\prime} / m^{\prime}$ & $\|$ & $\mathrm{cL}$ & cL6,2 & cL6,2 & $\mathrm{cC} 8,2$ \\
\hline & & $\stackrel{\perp}{\perp}$ & $\mathrm{cL}$ & a3,6 & a3,6 & $a 5,3$ \\
\hline \multirow[t]{3}{*}{67} & $6^{\prime} / m$ & $\|$ & $\mathrm{cL}$ & cL6,2 & cL6,2 & $\mathrm{cC} 8,2$ \\
\hline & & $\ddot{\perp}$ & $\mathrm{cL}$ & b5, 3 & b3,6 & $a, 6$ \\
\hline & & a.o. & b & a5, 6 & $a, 12$ & $\mathrm{a}, 12$ \\
\hline
\end{tabular}


TABLE II (cont.)

\begin{tabular}{|c|c|c|c|c|c|c|}
\hline (1) & $\overline{(\overline{(2)}}$ & (4) & $\overline{(5)}$ & $\overline{(6)}$ & $\overline{(7)}$ & $\overline{(\overline{(8)}}$ \\
\hline \multirow[t]{2}{*}{68} & $\overline{6} 1^{\prime}$ & 11 & $\mathrm{cL}$ & $\mathrm{cL} 6,2$ & cL6,2 & $\mathrm{cC} 8,2$ \\
\hline & & $\perp$ & cL & b3,6 & b5,3 & a, 6 \\
\hline \multirow[t]{3}{*}{69} & $6 / m 1^{\prime}$ & $\|$ & $\mathrm{cL}$ & cL6,2 & cL6,2 & $\mathrm{cC} 8,2$ \\
\hline & & $\perp$ & $\mathrm{cL}$ & b3,6 & $\mathrm{b} 3,6$ & $a, 6$ \\
\hline & & a.o. & b & a3,12 & a3,12 & $\mathrm{a}, 12$ \\
\hline \multirow[t]{2}{*}{70} & $4^{\prime} / m$ & $\|$ & $\mathrm{cL}$ & cL6,2 & cL6,2 & $c, 2$ \\
\hline & & $\underline{\perp}$ & cL & 63,4 & b3,4 & $a, 4$ \\
\hline \multirow[t]{2}{*}{71} & $4 / m 1^{\prime}$ & $\|$ & $c L$ & cL6,2 & cL6,2 & $\mathrm{cC} 8,2$ \\
\hline & & $\ddot{\perp}$ & $\mathrm{cL}$ & b3,4 & b3,4 & $\mathrm{a}, 4$ \\
\hline \multirow[t]{2}{*}{72} & $4^{\prime} / m^{\prime}$ & $\|$ & $c L$ & $\mathbf{c} 4,2$ & cL6,2 & $\mathrm{cC} 8,2$ \\
\hline & & $\perp$ & cL & $a, 4$ & $a, 4$ & $a, 4$ \\
\hline \multirow[t]{4}{*}{73} & $\overline{3} m$ & II & cL & cL6,2 & $\mathrm{cL} 6,2$ & $\mathrm{cC} 8,2$ \\
\hline & & $\stackrel{1}{1}$ & $\mathrm{cL}$ & c3,6 & $\mathrm{c} 3,6$ & c5,3 \\
\hline & & & $c L$ & $\mathrm{~b} 3,6$ & $\mathrm{~b} 3,6$ & $\mathrm{a}, 6$ \\
\hline & & & cL & a3,12 & $\mathrm{a} 3,12$ & a5,6 \\
\hline \multirow[t]{4}{*}{74} & $\overline{3}^{\prime} m$ & $\|$ & cL & cL9,1 & cL6,2 & $\mathrm{cC} 8,2$ \\
\hline & & $\perp$ & $\mathrm{cL}$ & b5,3 & b3,6 & a, 6 \\
\hline & & & $\mathrm{cL}$ & $a, 6$ & $a, 6$ & $c, 6$ \\
\hline & & & $\mathrm{cL}$ & $\mathrm{a} 5,6$ & $\mathrm{a}, 12$ & $\mathrm{a}, 12$ \\
\hline \multirow[t]{4}{*}{75} & $321^{\prime}$ & $\|$ & $c L$ & $\mathrm{cC} 8,2$ & $\mathrm{cL} 6,2$ & $\mathrm{cC} 8,2$ \\
\hline & & $\perp$ & cL & $c, 6$ & $c 5,3$ & $c, 6$ \\
\hline & & & $\mathrm{cL}$ & $a, 6$ & $a, 6$ & $\mathrm{a}, 6$ \\
\hline & & & $\mathrm{cL}$ & $\mathrm{a}, 12$ & a5,6 & $a, 12$ \\
\hline \multirow[t]{4}{*}{76} & $\overline{3} m 1^{\prime}$ & $\|$ & cL & cL6,2 & cL6,2 & $\mathrm{cC} 8,2$ \\
\hline & & $\perp$ & $\mathrm{cL}$ & $c 3,6$ & $\mathbf{c 3 , 6}$ & c, 6 \\
\hline & & & cL & $b 3,6$ & b3,6 & a, 6 \\
\hline & & & cL & a3,12 & $\mathrm{a} 3,12$ & $a, 12$ \\
\hline \multirow[t]{5}{*}{77} & $\overline{6} 2 m$ & $\|$ & $\mathrm{cL}$ & cL6,2 & cL6,2 & cC8,2 \\
\hline & & 1 & cL & cL5,3 & $\mathrm{cL} 5,3$ & c, 6 \\
\hline & & & cL & b3,6 & $\mathrm{b} 3,6$ & $c, 6$ \\
\hline & & & cL & b5,6 & b5,6 & $\mathrm{a}, 12$ \\
\hline & & a.o. & $\mathbf{b}$ & a5,12 & a5,12 & a5, 12 \\
\hline \multirow[t]{4}{*}{78} & $6221^{\prime}$ & $\|$ & cL & $\mathrm{cC} 8,2$ & cL6,2 & $\mathrm{cC} 8,2$ \\
\hline & & $\perp$ & $\mathrm{cL}$ & $c, 6$ & $\mathrm{c}, 6$ & c, 6 \\
\hline & & & $c L$ & $c, 6$ & $c, 6$ & c, 6 \\
\hline & & & cL & a,12 & a,12 & $\mathrm{a}, 12$ \\
\hline \multirow[t]{5}{*}{79} & $\overline{6} 2 m 1^{\prime}$ & $\|$ & $c L$ & cL6,2 & cL6,2 & $\mathrm{cC} 8,2$ \\
\hline & & $\because$ & cL & cL3,6 & cL5,3 & c, 6 \\
\hline & & & cI & b3,6 & $\mathrm{b} 3,6$ & c, 6 \\
\hline & & & $\mathrm{cL}$ & b3,12 & $\mathrm{b} 5,6$ & a,12 \\
\hline & & & $\mathrm{b}$ & $\mathrm{a}, 24$ & $\mathrm{a} 5,12$ & $\mathrm{a}, 24$ \\
\hline
\end{tabular}


TABLE II (cont.)

\begin{tabular}{|c|c|c|c|c|c|c|}
\hline$\overline{(1)}$ & (2) & (4) & (5) & $\overline{(6)}$ & (7) & (8) \\
\hline \multirow[t]{4}{*}{80} & $6^{\prime} 22^{\prime}$ & $\pi$ & $\overline{\mathrm{cL}}$ & $\mathrm{cC} 8,2$ & $\mathrm{cL} 6,2$ & $\mathrm{cC} 8,2$ \\
\hline & & $\perp$ & cL & $c 5,3$ & $c, 6$ & $c 5,3$ \\
\hline & & & cL & $a, 6$ & $a, 6$ & $a, 6$ \\
\hline & & & $\mathrm{cL}$ & a5,6 & $\mathrm{a}, 12$ & a5,6 \\
\hline \multirow[t]{5}{*}{81} & $6 / \mathrm{mmm}$ & $\|$ & $\mathrm{cL}$ & cL6,2 & cL6,2 & $\mathrm{cC} 8,2$ \\
\hline & & $\perp$ & $\mathrm{cL}$ & cL3,6 & cL3,6 & $\mathrm{c}, 6$ \\
\hline & & & cl & cL3,6 & cL3,6 & $c, 6$ \\
\hline & & & cL & $\mathrm{b} 3,12$ & b3,12 & $\mathrm{a}, 12$ \\
\hline & & a.o. & b & $\mathrm{a} 3,24$ & a3,24 & $a 5,12$ \\
\hline \multirow[t]{5}{*}{82} & $6 / m m m 1$ & $\|$ & cL & cL6,2 & cL6,2 & $\mathrm{cC} 8,2$ \\
\hline & & $\perp$ & $\mathrm{cL}$ & $\mathrm{cL} 3,6$ & cL3, 6 & $c, 6$ \\
\hline & & & $\mathrm{cL}$ & $\mathrm{cL3} 3,6$ & cL3, 6 & $\mathrm{c}, 6$ \\
\hline & & & $\mathrm{cL}$ & b3,12 & b3,12 & $\mathrm{a}, 12$ \\
\hline & & a.o. & $\mathrm{b}$ & $\mathrm{a} 3,24$ & a3,24 & $\mathrm{a}, 24$ \\
\hline \multirow[t]{4}{*}{83} & $\overline{6} ' m 2 '$ & $\|$ & $\mathrm{cL}$ & cL9,1 & cL6,2 & $\mathrm{cC} 8,2$ \\
\hline & & $\perp$ & $\mathrm{cL}$ & $\mathrm{b} 3,6$ & b5,3 & $a, 6$ \\
\hline & & & $c L$ & $a, 6$ & $a, 6$ & c5,3 \\
\hline & & & $\mathrm{cL}$ & $\mathrm{a}, 12$ & a5,6 & a5,6 \\
\hline \multirow[t]{4}{*}{84} & $6 / m^{\prime} m m$ & $\|$ & cL & cL9,1 & cL6,2 & cC8,2 \\
\hline & & $\perp$ & $\mathrm{cL}$ & $\mathrm{b} 3,6$ & b3,6 & $\mathrm{c}, 6$ \\
\hline & & & $\mathrm{cL}$ & b3,6 & b3,6 & $c, 6$ \\
\hline & & & $\mathrm{cL}$ & $\mathrm{a}, 12$ & $\mathrm{a}, 12$ & $\mathrm{a}, 12$ \\
\hline \multirow[t]{4}{*}{85} & $6^{\prime} / \mathrm{mmm}^{\prime}$ & $\|$ & cL & $\mathrm{cL} 6,2$ & cL6,2 & $\mathrm{cC} 8,2$ \\
\hline & & $\perp$ & cL & $\operatorname{cL5}, 3$ & cL3,6 & $c, 6$ \\
\hline & & & $\mathrm{cL}$ & $\mathrm{b} 3,6$ & b3,6 & $c, 6$ \\
\hline & & & $\mathrm{cL}$ & b5,6 & b3,12 & $\mathrm{a}, 12$ \\
\hline \multirow[t]{4}{*}{86} & $6^{\prime} / m^{\prime} m^{\prime} m$ & $\|$ & $\mathrm{cL}$ & $\mathrm{cL} 6,2$ & cL6,2 & $\mathrm{cC} 8,2$ \\
\hline & & $\perp$ & $\mathrm{cL}$ & $\mathrm{c} 3,6$ & $\mathrm{c} 3,6$ & c5,3 \\
\hline & & & $\mathrm{cL}$ & $\mathrm{b} 3,6$ & b3,6 & $a, 6$ \\
\hline & & & cL & $\mathrm{a} 3,12$ & $a 3,12$ & a5,6 \\
\hline \multirow[t]{4}{*}{87} & $4221 '$ & $\|$ & $\mathrm{cL}$ & $\mathrm{cC} 8,2$ & cL6,2 & $\mathrm{cC} 8,2$ \\
\hline & & $\perp$ & cL & $c, 4$ & $\mathrm{c}, 4$ & c, 4 \\
\hline & & & $\mathrm{cL}$ & $c, 4$ & c, 4 & $c, 4$ \\
\hline & & & $\mathrm{cL}$ & $a, 8$ & $a, 8$ & $a, 8$ \\
\hline \multirow[t]{4}{*}{88} & $4^{\prime} 22^{\prime}$ & $\|$ & $\mathrm{cL}$ & c,2 & cL6,2 & $\mathrm{c}, 2$ \\
\hline & & $\perp$ & $\mathrm{cL}$ & c,4 & $\mathrm{c}, 4$ & $c, 4$ \\
\hline & & & cL & $\mathrm{a}, 4$ & $\mathbf{a}, 4$ & $\mathbf{a}, 4$ \\
\hline & & & cL & $a, 8$ & $a, 8$ & a, \\
\hline
\end{tabular}


TABLE II (cont.)

\begin{tabular}{|c|c|c|c|c|c|c|}
\hline$(1)$ & (2) & (4) & (5) & (6) & (7) & $(8)$ \\
\hline \multirow[t]{5}{*}{89} & $4 / m m m$ & $\|$ & $\mathrm{cL}$ & cL6,2 & cL6,2 & $\mathrm{cC} 8,2$ \\
\hline & & $\perp$ & cL & cL3,4 & cL3,4 & $c, 4$ \\
\hline & & & cL & cL3,4 & cL3,4 & $c, 4$ \\
\hline & & & $\mathrm{cL}$ & b3,8 & b3,8 & $a, 8$ \\
\hline & & a.o. & b & $\mathrm{a} 3,16$ & a3,16 & $a 5,8$ \\
\hline \multirow[t]{5}{*}{90} & $4 / m m m 1^{\prime}$ & $\|$ & $\mathrm{cL}$ & cL6,2 & cL6,2 & $\mathrm{cC} 8,2$ \\
\hline & & $\ddot{\perp}$ & $\mathrm{cL}$ & cL3,4 & cL3,4 & $c, 4$ \\
\hline & & & $\mathrm{cL}$ & cL3,4 & cL3,4 & $c, 4$ \\
\hline & & & cL & b3,8 & b3,8 & $a, 8$ \\
\hline & & a.o. & b & a.3,16 & a3,16 & $a, 16$ \\
\hline \multirow[t]{4}{*}{91} & $4 / m^{\prime} m m$ & $\|$ & $\mathrm{cL}$ & $\operatorname{cL} 9,1$ & cL6,2 & $\mathrm{cC8} 8,2$ \\
\hline & & $\ddot{\perp}$ & $\mathrm{cL}$ & b3,4 & b3,4 & $c, 4$ \\
\hline & & & $c L$ & b3,4 & $\mathrm{b} 3,4$ & $c, 4$ \\
\hline & & & $\mathrm{cL}$ & $a, 8$ & $a, 8$ & $a, 8$ \\
\hline \multirow[t]{4}{*}{92} & $4^{\prime} / \mathrm{mmm}^{\prime}$ & $\|$ & $\mathrm{cL}$ & cL6,2 & cL6,2 & $\mathrm{c}, 2$ \\
\hline & & $\ddot{\perp}$ & $\mathrm{cL}$ & $\mathrm{cL3}, 4$ & cL3,4 & $c, 4$ \\
\hline & & & $\mathrm{cL}$ & b3,4 & $\mathrm{b} 3,4$ & $a, 4$ \\
\hline & & & $\mathrm{cL}$ & b3,8 & b3,8 & $a, 8$ \\
\hline \multirow[t]{4}{*}{93} & $4^{\prime} / m^{\prime} m^{\prime} m$ & $\|$ & cL & $\mathrm{cL} 4,2$ & cL6,2 & $\mathrm{cC8}, 2$ \\
\hline & & $\perp$ & cL & $\mathrm{c}, 4$ & $c, 4$ & $\mathrm{c}, 4$ \\
\hline & & & $\mathrm{cL}$ & b3,4 & $\mathrm{b3}, 4$ & $c, 4$ \\
\hline & & & $\mathrm{cL}$ & $a, 8$ & $a, 8$ & a, 8 \\
\hline \multirow[t]{2}{*}{94} & $6^{\prime}$ & $\|$ & cL & $\mathrm{cC} 8,2$ & cL9,1 & cC8,2 \\
\hline & & $\stackrel{\perp}{\perp}$ & $\mathrm{cL}$ & $a 5,3$ & $a, 6$ & a5,3 \\
\hline \multirow[t]{2}{*}{95} & 31' & $\|$ & $\mathrm{cL}$ & $\mathrm{cC} 8,2$ & cL9,1 & cC8,2 \\
\hline & & $\perp$ & $\mathrm{cL}$ & $a, 6$ & a5,3 & $a, 6$ \\
\hline \multirow[t]{2}{*}{96} & $61^{\prime}$ & $\|$ & $\mathrm{cL}$ & $\mathrm{cC} 8,2$ & cL9,1 & $\mathrm{cC} 8,2$ \\
\hline & & 1 & $\mathrm{cL}$ & $a, 6$ & $a, 6$ & $a, 6$ \\
\hline \multirow[t]{2}{*}{97} & $4^{\prime}$ & $\|$ & $\mathrm{cL}$ & $c, 2$ & cL9,1 & $c, 2$ \\
\hline & & $\perp$ & $\mathrm{cL}$ & $a, 4$ & $\mathbf{a}, 4$ & $a, 4$ \\
\hline \multirow[t]{2}{*}{98} & $41^{\prime}$ & $\|$ & $\mathrm{cL}$ & $\mathrm{cC} 8,2$ & cL 9,1 & $\mathrm{cC} 8,2$ \\
\hline & & $\stackrel{\perp}{ }$ & $\mathrm{cL}$ & $a, 4$ & $a, 4$ & $\mathrm{a}, 4$ \\
\hline \multirow[t]{4}{*}{99} & $3 m$ & $\|$ & $\mathrm{cL}$ & cL9,1 & $\operatorname{cL} 9,1$ & cC8,2 \\
\hline & & 1 & $\mathrm{cL}$ & $a, 6$ & $a, 6$ & $c 5,3$ \\
\hline & & & $\mathrm{cL}$ & b5,3 & b5,3 & a, 6 \\
\hline & & & $c L$ & $a 5,6$ & $a 5,6$ & $a 5,6$ \\
\hline \multirow[t]{4}{*}{100} & $3 m 1^{\prime}$ & $\|$ & cL & cL6,2 & cL9,1 & cC8,2 \\
\hline & & $\perp$ & $\mathrm{cL}$ & b3,6 & b5,3 & $a, 6$ \\
\hline & & & $\mathrm{cL}$ & $a, 6$ & $a, 6$ & c, 6 \\
\hline & & & $\mathrm{cL}$ & $a, 12$ & $\mathbf{a} 5,6$ & $a, 12$ \\
\hline
\end{tabular}


TABLE II (cont.)

\begin{tabular}{|c|c|c|c|c|c|c|}
\hline$(1)$ & (2) & (4) & (5) & (6) & (7) & (8) \\
\hline \multirow[t]{5}{*}{101} & $4 m m$ & $\pi$ & $\mathrm{cL}$ & cL 9,1 & cL 9,1 & $\mathrm{cC} 8,2$ \\
\hline & & $\perp$ & cL & b3,4 & b3,4 & $c, 4$ \\
\hline & & & cL & b3,4 & b3,4 & $c, 4$ \\
\hline & & & $\mathrm{cL}$ & $a, 8$ & $a, 8$ & $a, 8$ \\
\hline & & a.o. & b & $\mathrm{a} 5,8$ & $a 5,8$ & $a 5,8$ \\
\hline \multirow[t]{5}{*}{102} & $4 m m 1^{\prime}$ & $\|$ & $\mathrm{cL}$ & cL6,2 & cL 9,1 & cC8,2 \\
\hline & & $\perp$ & $\mathrm{cL}$ & b3,4 & b3,4 & $c, 4$ \\
\hline & & & $\mathrm{cL}$ & b3,4 & b3,4 & $c, 4$ \\
\hline & & & $\mathrm{cL}$ & $a, 8$ & $a, 8$ & $a, 8$ \\
\hline & & a.o. & $\mathbf{b}$ & $\mathrm{a}, 16$ & a5,8 & $a, 16$ \\
\hline \multirow[t]{4}{*}{103} & $4^{\prime} m m^{\prime}$ & $\|$ & cL & cL 4,2 & cL9,1 & c, 2 \\
\hline & & 1 & cL & b3,4 & b3,4 & $c, 4$ \\
\hline & & & $\mathrm{cL}$ & $a, 4$ & $a, 4$ & $\mathrm{a}, 4$ \\
\hline & & & $\mathrm{cL}$ & $\mathrm{a}, 8$ & a, 8 & $a, 8$ \\
\hline \multirow[t]{4}{*}{104} & $6^{\prime} \mathrm{mm}$ & $\|$ & $\mathrm{cL}$ & cL6,2 & cL9,1 & $\mathrm{cC} 8,2$ \\
\hline & & $\perp$ & $\mathrm{cL}$ & b5,3 & b3,6 & $a, 6$ \\
\hline & & & $\mathrm{cL}$ & $a, 6$ & $a, 6$ & $c 5,3$ \\
\hline & & & $\mathrm{cL}$ & $a 5,6$ & $a, 12$ & $a 5,6$ \\
\hline \multirow[t]{5}{*}{105} & $6 m m$ & $\|$ & $\mathrm{cL}$ & cL9,1 & $\operatorname{cL} 9,1$ & $\mathrm{cC} 8,2$ \\
\hline & & $\stackrel{\perp}{\perp}$ & $\mathrm{cL}$ & b3, 6 & $\mathrm{~b} 3,6$ & $c, 6$ \\
\hline & & & $\mathrm{cL}$ & b3, 6 & b3,6 & c, 6 \\
\hline & & & $\mathrm{cL}$ & $\mathrm{a}, 12$ & $\mathrm{a}, 12$ & $\mathrm{a}, 12$ \\
\hline & & a.o. & b & $\mathrm{a} 5,12$ & $\mathrm{a} 5,12$ & $a 5,12$ \\
\hline \multirow[t]{5}{*}{106} & $6 m m 1^{\prime}$ & \|ा & cL & cL6,2 & cL9,1 & $\mathrm{cC} 8,2$ \\
\hline & & $\perp$ & cL & b3,6 & b3, 6 & $c, 6$ \\
\hline & & & $\mathrm{cL}$ & b3,6 & b3,6 & $c, 6$ \\
\hline & & & cL & $\mathrm{a}, 12$ & $a, 12$ & $a, 12$ \\
\hline & & a.o. & b & $a, 24$ & a5,12 & $a, 24$ \\
\hline
\end{tabular}


TABLE III

The characteristic optical effects of the isotropic (cubic) crystals in the fields $\boldsymbol{F}=k, E, H .{ }^{*}$ (For abbreviations see the text).

\begin{tabular}{|c|c|c|c|c|c|}
\hline \multirow[b]{2}{*}{ No } & \multirow[b]{2}{*}{$K$} & \multirow[b]{2}{*}{$s$} & \multicolumn{3}{|c|}{ The effects for $F=$} \\
\hline & & & $\boldsymbol{k}$ & $\boldsymbol{E}$ & $\boldsymbol{H}$ \\
\hline (1) & $(2)$ & $(4)$ & $(6)$ & (7) & $(8)$ \\
\hline \multirow[t]{5}{*}{107} & 23 & $\| z$ & $\mathrm{c}, 2$ & $c, 2$ & $\mathrm{c}, 2$ \\
\hline & & 1 & $c, 4$ & $c, 4$ & $\mathrm{c}, 4$ \\
\hline & & & $a, 4$ & $\mathrm{a}, 4$ & $a, 4$ \\
\hline & & $\| d$ & $\mathrm{cC} 5,1$ & cC5,1 & cC5, 1 \\
\hline & & 1 & $\mathrm{a} 5,3$ & $\mathrm{a} 5,3$ & a5,3 \\
\hline \multirow[t]{5}{*}{108} & $m^{\prime} 3$ & $\| z$ & $\mathrm{c}, 2$ & $c, 2$ & c, 2 \\
\hline & & $\perp$ & $c, 4$ & $c, 4$ & $c, 4$ \\
\hline & & & $a, 4$ & $\mathrm{a}, 4$ & $a, 4$ \\
\hline & & $\| d$ & cL 9,1 & $\mathrm{cC} 8,2$ & $\mathrm{cC} 8,2$ \\
\hline & & $\perp$ & a5,3 & $a, 6$ & a,6 \\
\hline \multirow[t]{5}{*}{109} & $231^{\prime}$ & $\| z$ & $\mathrm{c}, 2$ & $c, 2$ & $\mathrm{c}, 2$ \\
\hline & & $\perp$ & $c, 4$ & $c, 4$ & $\mathrm{c}, 4$ \\
\hline & & & $a, 4$ & $\mathrm{a}, 4$ & $a, 4$ \\
\hline & & $\| d$ & $\mathrm{cC} 8,2$ & cL 9,1 & $\mathrm{cC} 8,2$ \\
\hline & & $\perp$ & $a, 6$ & $\mathrm{a} 5,3$ & a, 6 \\
\hline \multirow[t]{8}{*}{110} & $\overline{4} 3 m^{\prime}$ & $\| z$ & $\mathrm{cL} 6,2$ & $c, 2$. & $c, 2$ \\
\hline & & $\perp$ & cL 6,4 & $c, 4$ & $c, 4$ \\
\hline & & & $a, 4$ & $a, 4$ & $a, 4$ \\
\hline & & & $a, 8$ & $a, 8$ & $a, 8$ \\
\hline & & $\| d$ & cC7,2 & $\mathrm{cC} 5,1$ & cC5,1 \\
\hline & & 1 & a5,3 & $a, 6$ & $a, 6$ \\
\hline & & & $a, 6$ & a5,3 & a5,3 \\
\hline & & & 25,6 & $a 5,6$ & a5,6 \\
\hline \multirow[t]{5}{*}{111} & $m 3$ & $\| z$ & cL3,2 & cL3,2 & $c, 2$ \\
\hline & & $\perp$ & $\mathrm{cL} 3,4$ & $\mathrm{cL} 3,4$ & $c, 4$ \\
\hline & & & $\mathrm{b} 3,4$ & b3,4 & $a, 4$ \\
\hline & & $\| d$ & $\mathrm{cC} 7,2$ & $\mathrm{cC} 7,2$ & cC5,1 \\
\hline & & $\perp$ & a3,6 & a3,6 & a5,3 \\
\hline \multirow[t]{5}{*}{112} & $m 31^{\prime}$ & $\| z$ & $\mathrm{cL} 3,2$ & cL3,2 & $c, 2$ \\
\hline & & $\perp$ & cL3,4 & cL3,4 & $\mathrm{c}, 4$ \\
\hline & & & $\mathrm{b} 3,4$ & b3,4 & $a, 4$ \\
\hline & & $\| d$ & cL6,2 & cL6,2 & cC8,2 \\
\hline & & $\perp$ & $\mathrm{a} 3,6$ & a3,6 & a,6 \\
\hline
\end{tabular}

*The results for $\boldsymbol{F}=0$ are omitted due to the fact that for all directions of 8 in the crystals we have one type of waves, $\mathrm{cL}$, i.e. two transverse linearly polarized waves. 
TABLE III (cont.)

\begin{tabular}{|c|c|c|c|c|c|}
\hline$(1)$ & (2) & (4) & (6) & (7) & (8) \\
\hline \multirow[t]{8}{*}{113} & \multirow[t]{8}{*}{$43 m$} & $z$ & cL 4,2 & cL4,2 & $\mathrm{cC} 8,2$ \\
\hline & & 1 & cL 4,4 & cL 4,4 & $\mathrm{cC} 8,4$ \\
\hline & & & $\mathrm{b} 3,4$ & b3,4 & $c, 4$ \\
\hline & & & $a, 8$ & $a, 8$ & a, 8 \\
\hline & & $\| d$ & cL 9,1 & cL 9,1 & cC8,2 \\
\hline & & $\perp$ & $a, 6$ & $a, 6$ & $a 5,3$ \\
\hline & & & b5,3 & b5,3 & $a, 6$ \\
\hline & & & $\mathrm{a} 5,6$ & $\mathrm{a} 5,6$ & a5,6 \\
\hline \multirow[t]{8}{*}{114} & \multirow[t]{8}{*}{$m^{\prime} 3 m$} & $\| z$ & cL 4,2 & cL6,2 & $\mathrm{cC} 8,2$ \\
\hline & & 1 & $\mathrm{cL} 4,4$ & cL 6,4 & cC8,4 \\
\hline & & & $\mathrm{b} 3,4$ & $\mathrm{~b} 3,4$ & c, 4 \\
\hline & & & $a, 8$ & $a, 8$ & $a, 8$ \\
\hline & & $\| d$ & cLL9,1 & cL6,2 & cC8,2 \\
\hline & & $\perp$ & $a, 6$ & $a, 6$ & $\mathrm{c}, 6$ \\
\hline & & & $\mathrm{b} 5,3$ & b3,6 & $a 5,3$ \\
\hline & & & $a 5,6$ & $\mathrm{a}, 12$ & $\mathrm{a}, 12$ \\
\hline \multirow[t]{8}{*}{115} & \multirow[t]{8}{*}{$\overline{4} 3 m 1^{\prime}$} & $\| z$ & cL6,2 & cL4,2 & $\mathrm{cC} 8,2$ \\
\hline & & $\perp$ & cL 6,4 & $\operatorname{cL} 4,4$ & cC8,2 \\
\hline & & & b3,4 & b3,4 & c, 4 \\
\hline & & & $a, 8$ & $a, 8$ & a, 8 \\
\hline & & $\| d$ & cL 6,2 & cL 9,1 & $\mathrm{cC} 8,2$ \\
\hline & & $\perp$ & $a, 6$ & $a, 6$ & a, 6 \\
\hline & & & b3,6 & b5,3 & $a, 6$ \\
\hline & & & $a, 12$ & $a 5,6$ & $a, 12$ \\
\hline \multirow[t]{8}{*}{116} & \multirow[t]{8}{*}{432} & $\| z$ & $\mathrm{cC} 8,2$ & $\mathrm{cC} 8,2$ & $\mathrm{cC} 8,2$ \\
\hline & & $\perp$ & cC8,4 & $\mathrm{cC} 8,4$ & cC8,4 \\
\hline & & & $c, 4$ & $c, 4$ & $c, 4$ \\
\hline & & & $a, 8$ & $a, 8$ & $a, 8$ \\
\hline & & $\| d$ & $\mathrm{cC} 8,2$ & $\mathrm{cC} 8,2$ & cC8,2 \\
\hline & & $\perp$ & c5,3 & $c 5,3$ & c5,3 \\
\hline & & & $a, 6$ & $a, 6$ & $a, 6$ \\
\hline & & & a5,6 & $\mathrm{a} 5,6$ & $a 5,6$ \\
\hline \multirow[t]{8}{*}{117} & \multirow[t]{8}{*}{$m^{\prime} 3 m^{\prime}$} & $\| z$ & $\mathrm{cL} 6,2$ & $\mathrm{cC} 8,2$ & $\mathrm{cC} 8,2$ \\
\hline & & 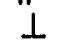 & cL 6,4 & $\mathrm{cC} 8,4$ & $\mathrm{cC} 8,4$ \\
\hline & & & $c, 4$ & $c, 4$ & $c, 4$ \\
\hline & & & $a, 8$ & $a, 8$ & $a, 8$ \\
\hline & & $\| d$ & cL 6,2 & $\mathrm{cC} 8,2$ & $\mathrm{cC} 8,2$ \\
\hline & & $\perp$ & c5,3 & $c, 6$ & $c, 6$ \\
\hline & & & $a, 6$ & $a, 6$ & $a, 6$ \\
\hline & & & $a 5,6$ & a,12 & a,12 \\
\hline
\end{tabular}


TABLE III (cont.)

\begin{tabular}{|c|c|c|c|c|c|}
\hline (1) & $\overline{\overline{(2)}}$ & $\overline{(4)}$ & $\overline{(6)}$ & $(7)$ & $\overline{(8)}$ \\
\hline \multirow[t]{8}{*}{118} & $4321^{\prime}$ & $\| z$ & $\mathrm{cCC} 8,2$ & $\mathrm{cL} 6,2$ & $\mathrm{cCC} 8,2$ \\
\hline & & $\perp$ & $\mathrm{cC} 8,4$ & cL6,4 & $\mathrm{cC} 8,4$ \\
\hline & & & c, 4 & c, 4 & $c, 4$ \\
\hline & & & $a, 8$ & $a, 8$ & $a, 8$ \\
\hline & & $\| d$ & $\mathrm{cC} 8,2$ & cL6,2 & $\mathrm{cC} 8,2$ \\
\hline & & $\perp$ & $c, 6$ & $c 5,3$ & c, 6 \\
\hline & & & $a, 6$ & $a, 6$ & $a, 6$ \\
\hline & & & $\mathrm{a}, 12$ & a5,6 & $a, 12$ \\
\hline \multirow[t]{8}{*}{119} & $4^{\prime} 32^{\prime}$ & $\| z$ & c,2 & cL6,2 & $c, 2$ \\
\hline & & $\perp$ & $c, 4$ & cL6,4 & $c, 4$ \\
\hline & & & $a, 4$ & $\mathrm{a}, 4$ & $a, 4$ \\
\hline & & & $a, 8$ & $a, 8$ & $a, 8$ \\
\hline & & $\| d$ & cC5,1 & $\mathrm{cC} 7,2$ & cC5,1 \\
\hline & & $\perp$ & $\mathrm{a} 5,3$ & $a, 6$ & $\mathrm{a} 5,3$ \\
\hline & & & $a, 6$ & a5,3 & $a, 6$ \\
\hline & & & $\mathrm{a} 5,6$ & $\mathrm{a} 5,6$ & a5,6 \\
\hline \multirow[t]{8}{*}{120} & $m 3 m^{\prime}$ & $\| z$ & cL6,2 & cL6,2 & $\mathrm{c}, 2$ \\
\hline & & $\perp$ & cL6,4 & cL6,4 & $c, 4$ \\
\hline & & & b3,4 & b3,4 & $a, 4$ \\
\hline & & & b3,8 & b3,8 & $a, 8$ \\
\hline & & $\| d$ & $\mathrm{cC} 7,2$ & $\mathrm{cC} 7,2$ & cC5,1 \\
\hline & & $\perp$ & $a 3,6$ & $\mathrm{a} 3,6$ & $a, 6$ \\
\hline & & & $\mathrm{a} 3,12$ & a3,12 & $\mathrm{a} 5,6$ \\
\hline & & & a3,6 & $\mathrm{a} 3,6$ & a5,3 \\
\hline \multirow[t]{8}{*}{121} & $m 3 m$ & $\| z$ & $\mathrm{cL} 6,2$ & cL6,2 & $\mathrm{cC} 8,2$ \\
\hline & & $\perp$ & cL6,4 & cL6,4 & $\mathrm{cC} 8,4$ \\
\hline & & & cL3,4 & cL3,4 & $c, 4$ \\
\hline & & & b3,8 & b3,8 & a, 8 \\
\hline & & $\| d$ & $\mathrm{cL} 6,2$ & cL6,2 & $\mathrm{cC} 8,2$ \\
\hline & & $\perp$ & c3,6 & c3,6 & $c 5,3$ \\
\hline & & & b3,6 & b3,6 & $a, 6$ \\
\hline & & & a3,12 & a3,12 & $\mathrm{a} 5,6$ \\
\hline \multirow{8}{*}{122} & $m 3 m 1^{\prime}$ & $\| z$ & $\mathrm{cL} 6,2$ & cL6,2 & $\mathrm{cC} 8,2$ \\
\hline & & $\perp$ & $\mathrm{cL} 6,4$ & cL6,4 & $\mathrm{cC} 8,4$ \\
\hline & & & cL3,4 & cL3,4 & $\mathrm{c}, 4$ \\
\hline & & & b3,8 & b3,8 & $a, 8$ \\
\hline & & $\| d$ & cL6,2 & $\mathrm{cL} 6,2$ & $\mathrm{cC} 8,2$ \\
\hline & & $\perp$ & $\mathrm{c} 3,6$ & c3,6 & $c, 6$ \\
\hline & & & b3,6 & b3 6 & $a, 6$ \\
\hline & & & a3,12 & a3,12 & $\mathrm{a}, 12$ \\
\hline
\end{tabular}




\section{Acknowledgments}

It is a pleasure to thank Drs. P. Kosiński and M. Majewski for stimulating discussions and for helpful remarks on the manuscript.

\section{References}

[1]. C.J. Bradley, A.P. Cracknell, The Mathematical Theory of Symmetry in Solids, Clarendon Press, Oxford 1972.

[2] V.A. Agranovich, V.K. Ginzburg, Kristallooptika z uchetom prostranstvennoi dispersii i teoria eksitonov, Nauka, Moskva 1979 (in Russian).

[3] S. Malinowski, Acta Phys. Pol. A79, 565 (1991).

[4] M. Born, E. Wolf, Principles of Optics, MacMillan, New York 1959.

[5] K. Vainshtein, A.A. Chernov, L.A. Shuvalov (eds.), The Modern Crystallography Vol. 1-4, Nauka, Moskva 1979 (in Russian).

[6] S. Malinowski, Acta Phys. Pol. A77, 543 (1990).

[7] J.P. Jan, Solid State Phys. 5, 1 (1957).

[8] S. Malinowski, Acta Phys. Pol. A78, 921 (1990).

[9] V.V. Eremenko, N.F. Kharchenko, Yu.G. Litvinenko, V.M. Naumenko, Magnietooptika i spektroskopia antiferromagnetikov, Dumka, Kiev 1989 (in Russian).

[10] J. Ferr'e, G.A. Gehring, Rep. Prog. Phys. 47, 513 (1984). 